\title{
SREATE
}

Centre de Recherche en économie de l'Environnement, de l'Agroalimentaire, des Transports et de l'Énergie
Center for Research on the economics of the Environment, Agri-food, Transports and Energy

\section{Innovation and Antibiotic Use within Antibiotic Classes: Market Incentives and Economic Instruments}

\author{
Markus Herrmann \\ Bruno Nkuiya \\ Anne-Renée Dussault
}

Cahier de recherche/Working Paper 2013-3

Mai/May 2013

Herrmann: Corresponding author. Department of Economics, CREATE, Pavillon J.-A.-DeSève, 1025, av. des Sciences-Humaines, Université Laval, Québec, Canada G1V 0A6

markus.herrmann@ecn.ulaval.ca

Nkuiya: Department of Economics, CREATE, Pavillon J.-A.-DeSève, 1025, av. des Sciences-Humaines, Université Laval, Québec, Canada G1V OA6

Dussault: Conseil du Trésor du Québec

We wish to thank participants of the conference in honour of Gérard Gaudet "Environmental and Resource Economics - 80 years after Hotelling". Financial support was granted by the Fonds québécois de la recherche sur la société et la culture.

Les cahiers de recherche du CREATE ne font pas l'objet d'un processus d'évaluation par les pairs/CREATE working papers do not undergo a peer review process.

ISSN 1927-5544 


\begin{abstract}
:
We analyze a monopolist's incentive to innovate a new antibiotic which is connected to the same pool of antibiotic treatment efficacy as is another drug produced by a generic industry. We outline the differences of antibiotic use under market conditions and in the social optimum. A time and state-dependent tax-subsidy mechanism is proposed to induce the monopolist and generic industry to exploit antibiotic efficacy optimally.
\end{abstract}

Keywords: Economics of antibiotic resistance, antibiotic innovation, monopoly, generic industry, social optimum, economic instruments

Classification JEL: D21, D42, I18, Q38 
"The message on this World Health Day is loud and clear. The world is on the brink of losing these miracle cures ... In the absence of urgent corrective and protective actions, the world is heading towards a post-antibiotic era, in which many common infections will no longer have a cure and, once again, kill unabated."

Dr Margaret Chan, director-general of the World Health Organzation, on the occasion of the World Health Day, April 7, 2011

\section{Introduction}

Bacterial resistance has become a major problem in hospital and outpatient environments, rendering existing antibiotic treatments less effective or even futile. Within its multiple causes, one may refer to the misuse by patients and, what can be called, the overuse within the community. More than "80 years after Hotelling," economists now consider antibiotic treatment efficacy, the mirror image of antibiotic resistance, as a valuable, scarce, biological resource, which merits attention.

In the past, the pharmaceutical industry mostly kept pace with the rise of bacterial resistance by introducing new antibiotics - so called analogues - which belonged to already existing antibiotic classes (e.g. the penicillins form one class among the 15 existing antibiotic classes). The development of completely new antibiotic classes has been considerably more costly and has failed in many cases because of their toxicity to humans (Coates et al., 2011). These authors also report on a study by Becker et al. (2006), which suggests that no new classes can be developed in the future, as all "broad-spectrum antibacterials have been discovered (page 191)". This may explain why the pharmaceutical industry currently concentrates on the development of analogues. However, there seems to exist a limit "to the number of analogues which can be made from a single chemical core ... and eventually, 
bacteria can evolve resistance beyond the scope of even the most ingenious medicinal chemist (page 187)", which implies that all analogues of a given class will eventually lose their treatment effectiveness.

This paper addresses the incentives of a firm to innovate a new antibiotic analogue within a given antibiotic class. We assume that all antibiotic analogues are linked to a common pool of treatment effectiveness in the sense that their use causes the common pool to decline. The innovating firm thus faces an externality as the use of antibiotics within the antibiotic class impacts on the treatment effectiveness of the class in general, and on its own antibiotic in particular. Furthermore, we make the simplifying assumption that the new antibiotic analogue targets a distinct market from the one served by the existing antibiotic class such that antibiotic analogues do not represent substitutes in fighting an infectious disease. While the innovating firm is granted a patent of limited lifetime in its market, the other market is assumed to be served by a generic industry which behaves as having open access to the common resource pool of antibiotic treatment effectiveness.

A related example is that of the Quinolones antibiotic class, antibiotics of which serve to fight human infections and, in the past, were often used to enhance growth of animals by preventing infection within the livestock. Scientific evidence showed that the intake of growth-promoted meat could enhance bacterial resistance in humans (Nelson et al., 2007), suggesting that antibiotics belonging to the same class were linked to a common pool of antibiotic efficacy. The fact that firms selling an antibiotic do not have an incentive to account for this externality has lead to the question on "How broad should the scope of patents be" (Laxminarayan 2002)? ${ }^{1}$

We show that the monopolist attaches an implicit value to the antibiotic treatment efficacy which corresponds to the intertemporal sum, properly discounted, of the treatment

\footnotetext{
${ }^{1}$ In 2005, the US Food and Drug Administration withdrew the Quinolone Baytril (produced by Bayer) for use in poultry water in order to prevent the spread of fluoroquinolone-resistant infections in humans (FDA 2005). The active antibacterial ingredient of Baytril is Enrofloxacin, which is also distributed by multiple generic producers in the European Union (for generic versions sold in EU member states, see Annex 1 to EU Directive 2001/82EC). Any development of a new fluoroquinolone antibiotic for human use earning a patent would thus potentially be affected by the use of Enrofloxacin in the generic market for animal use.
} 
efficacy's marginal profitability. The higher the endogenously determined relative impact of the generic industry on the common resource pool, the heavier the monopolist discounts future profits. The monopolist's incentive to incur a higher innovation cost is determined by the marginal avoided impact of the generic industry's on the common resource pool of treatment efficacy. While the monopolist accounts only for the impact of sharing the common resource on his own profits, the social optimum accounts for the social welfare generated in both markets. We determine the effects driving the order of the socially optimal use of antibiotics and present an efficiency-inducing tax-subsidy mechanism for both markets, operated by the monopolist and the generic industry. In the case that the regulator cannot commit to a time-dependent correcting policy, the monopolistic firm may turn that policy to its advantage. This points to the implementation of a "subgame-perfect," state-dependent mechanism, which we also characterize.

The socially optimal use of antibiotics when their treatment efficacy is non-renewable has been modeled by Laxminarayan and Brown (2001). Wilen and Msangi (2003) extend the analysis to the case of renewable antibiotic efficacy which gives rise to the existence of a steady state, where antibiotic efficacy is not completely exhausted. The market outcome and impact on treatment effectiveness have been modeled by Mechoulan (2007), Fischer and Laxminarayan (2004), Herrmann and Gaudet (2009) and Herrmann (2010). Fischer and Laxminarayan (2005) consider the sequential development of new antibiotics with exhaustible efficacy by a monopolistic firm. Rudholm (2002) characterizes antibiotic resistance spillover across countries and proposes a correcting, dynamic Piouvian tax. See Herrmann and Laxminarayan (2010) for a detailed review on the economics of antibiotic resistance.

This paper is structured as follows. Section 2 presents the model and proposes the analysis of the monopolist's as well as socially optimal incentives for antibiotic innovation and use. Section 3 compares the outcomes and addresses economic instruments which are susceptible to correct the industry's suboptimal behavior. We conclude in section 4 . 


\section{Model}

We propose a model in which two antibiotics $i=A, B$ belong to the same antibiotic class and are attached to a common pool of treatment efficacy. Use of antibiotic $i$ at time $t$ is denoted $f_{i}(t)$ and decreases the common level of the antibiotic class's efficacy $w(t)$. The distance in the biological formulas of the two antibiotics is measured by the parameter $\alpha$, which translates also the pressure that antibiotic $B$ exerts on the common pool of antibiotic efficacy relative to antibiotic $A$. The law of motion of the antibiotic class's efficacy can then be written as:

$$
\dot{w}(t)=-f_{A}(t)-\alpha f_{B}(t),
$$

which implies that antibiotic efficacy is modeled as a non-renewable resource affected linearly by the antibiotic use made in each market. We assume that parameter $\alpha$ satisfies $\alpha \geq 0$. When $\alpha=0$, the antibiotic class has only one member (antibiotic $A$ ), and $\alpha=1$ implies that the two antibiotics affect efficacy equally.

Each antibiotic is produced at a constant unit $\operatorname{cost} c_{i}$ and is sold on a different market. While market $B$ is assumed to be served by a generic industry, market $A$ is served by a monopolistic firm, once innovation has occured. We follow Herrmann and Gaudet (2009) and define a linear, inverse demand function for market $i=A, B$ as:

$$
P_{i}\left(f_{i}(t), w(t)\right)=r_{i} w(t)\left(1-f_{i}(t)\right),
$$

which has the choke price $r_{i} w(t)$ at time $t .^{2}$ The parameter $r_{i}$ is demand specific and relates to the willingness to pay in each market which evolves in conjunction with the treatment efficacy $w(t)$.

\footnotetext{
${ }^{2}$ This inverse demand function can be motivated on the grounds of utility maximization of an infected individual, who attributes a probability that the antibiotic will help him recover from infection. See Herrmann and Gaudet (2009). Note that life-threatening conditions may be better modeled with a demand function that does not have a choke price.
} 


\subsection{Monopoly and generic industry}

Economic incentives which characterize the antibiotic use made of each drug depend on the market structure occurring in markets $A$ and $B$. We assume that a generic industry currently serves market $B$ and will do so once the patent has expired and the monopolistic firm has become a member of a generic industry in market A. In particular, we model the firms of the generic industry as having open access to the common resource pool $w(t)$. These firms do not interact strategically and are not able to account for their impact of current antibiotic use on future levels of the antibiotic class's efficacy. In equilibrium, all economic rents are dissipated, such that the price in market $i$ is equal to the average production cost, i.e.:

$$
P_{i}\left(f_{i}(t), w(t)\right)=c_{i}
$$

where $c_{i}>0$ is the constant average production cost. ${ }^{3}$ Making use of our specification of the price function in (1), the quantity $f_{i}$ served in market $i$ can be determined from equilibrium condition (2) as:

$$
f_{i}(w(t))=\max \left\{1-\frac{c_{i}}{r_{i} w(t)}, 0\right\} .
$$

As long as $f_{i}>0$, drug $i$ is said to be economically viable in market $i$, which is assured as long as the level of antibiotic efficacy satisfies $w(t)>c_{i} / r_{i}$.

While the equilibrium condition (and dynamics) of a generic industry can be determined in a straightforward manner, modeling the intertemporal profit-maximizing behavior of a monopolist resides in a formulation using optimal control techniques, to which we now turn. We assume that the monopolist chooses initially the biological distance $\alpha$ of his $\operatorname{drug} A$ with respect to the existing $\operatorname{drug} B$. The controllable parameter $\alpha$ must satisfy $0 \leq \alpha \leq \bar{\alpha}$, where $\bar{\alpha}$ corresponds to the smallest distance between the two antibiotics warranting the creation of a patent. The innovation cost is given by $C(\alpha)$ with $C(0)=\infty, C^{\prime}(\alpha)<0$ and $C^{\prime \prime}(\alpha)>0$, where $C(0)=\infty$ precludes the development of a new antibiotic class, which will be assumed in what follows. Having entered market $A$, the monopolist decides on the quantity $f_{A}(t)$ as

\footnotetext{
${ }^{3}$ See Herrmann and Gaudet (2009) for a detailed analysis of this market type.
} 
long as the patent has not expired, which occurs at time $T$. We write the instantaneous profit function as $\pi\left(f_{A}(t), w(t)\right)=\left(P_{A}\left(f_{A}(t), w(t)\right)-c_{A}\right) f_{A}(t)$, and the intertemporal objective as:

$$
\begin{aligned}
\max _{\left\{\alpha, f_{A}(t)\right\}} \Pi & =\int_{0}^{T} e^{-\delta t} \pi\left(w(t), f_{A}(t)\right) d t-C(\alpha)+e^{-\delta T} V_{A}^{o a}(w(T)) \\
\text { s.t } \quad \dot{w}(t) & =-f_{A}-\alpha f_{B}(w(t)), \quad w(0)=w_{0}, \quad w(T) \geq 0 \\
0 & \leq \alpha \leq \bar{\alpha}
\end{aligned}
$$

where $\delta$ is the monopolist's discount rate and $V_{A}^{o a}$ is the monopolist's residual value, properly discounted, once the patent has expired and the monopolist has become a member of a generic industry. As, by assumption, a generic industry behaves blackas in market $B$ like having open access to the common resource pool, all economic rents are dissipated beyond time $T$ and we have $V_{A}^{o a} \equiv 0 .^{4}$

We write the current-value Hamiltonian

$$
H(\cdot)=\left[P\left(f_{A}(t), w(t)\right)-c_{A}\right] f_{A}-\lambda(t)\left[f_{A}(t)+\alpha f_{B}(w(t))\right]
$$

where $\lambda(t)$ is the shadow price associated to the use of the antibiotic class's efficacy. Necessary conditions for an optimum are

$$
\begin{aligned}
f_{A} & \geq 0, \frac{\partial H}{\partial f_{A}} \leq 0, \frac{\partial H}{\partial f_{A}} f_{A}=0 \\
f_{A} & \leq 1, \frac{\partial H}{\partial f_{A}} \geq 0, \frac{\partial H}{\partial f_{A}}\left(1-f_{A}\right)=0 \\
\dot{\lambda}-\delta \lambda & =-\frac{\partial H}{\partial w} \\
\dot{w} & =-f_{A}-\alpha f_{B}
\end{aligned}
$$

where $\frac{\partial H}{\partial f_{A}}=r_{A} w\left(1-2 f_{A}\right)-c_{A}-\lambda$ and $\frac{\partial H}{\partial w}=\frac{\partial \pi}{\partial w}-\alpha \lambda \frac{\partial f_{B}}{\partial w}$, as well as the condition for the controllable parameter ${ }^{5}$

$$
\alpha \leq \bar{\alpha}, \quad(\bar{\alpha}-\alpha)\left(\int_{0}^{T} e^{-\delta t} \frac{\partial H}{\partial \alpha} d t-\frac{\partial C}{\partial \alpha}\right)=0, \quad-\frac{\partial C}{\partial \alpha}+\int_{0}^{T} e^{-\delta t} \frac{\partial H}{\partial \alpha} d t \geq 0 .
$$

\footnotetext{
${ }^{4}$ We abstract for now from the possibility that a monopolist does not invest to develop a new drug. The condition for this to hold will be specified later.

${ }^{5}$ See theorem 7.11 .1 of Léonard and Long (1992) for this condition.
} 
and the transversality condition

$$
w(T) \geq 0, \quad \lambda(T) \geq 0 \quad \text { and } \quad w(T) \lambda(T)=0 .
$$

Note that it is not economically viable in any market to extract antibiotic efficacy completely as $c_{i}>0, i=A, B$. It follows that we must have $w(T)>0$, such that $\lambda(T)=0$.

Conditions (6) and (7) represent the Kuhn-Tucker conditions which guarantee the static efficiency of the monopolist's choice of the quantity sold. For an interior solution $0<f_{A}<1$, it reads as $r_{A} w\left(1-2 f_{A}\right)=c_{A}+\lambda$, i.e. the marginal revenue from selling antibiotic $A$ must equal the total marginal cost of doing so, which comprises the marginal production cost and the implicit value associated to antibiotic efficacy.

Condition (8) assures the dynamic efficiency of the monopolist's program. It represents a differential equation in $\lambda$ which can be rewritten as $\dot{\lambda}(t)-\beta(t) \lambda(t)=-\frac{\partial \pi}{\partial w}$, where we have defined $\beta(t) \equiv\left[\delta+\alpha \partial f_{B} / \partial w\right]$. Multiplying this equation by the integration factor $I(t)=$ $\exp \int_{t}^{T} \beta(s) d s$ and integrating both sides, we obtain the expression $\lambda(t) I(t)=\int_{t}^{T} \frac{\partial \pi}{\partial w} I(s) d s+$ $I(T) \lambda(T)$, from which we calculate:

$$
\lambda(t)=\int_{t}^{T} \frac{\partial \pi}{\partial w} \exp \left(-\int_{t}^{s} \beta(\tau) d \tau\right) d s
$$

where we have used the boundary condition $\lambda(T)=0$ in order to obtain the last equality. The implicit value $\lambda(t)$ thus corresponds to the intertemporal sum, "properly" discounted of the marginal profitability of antibiotic efficacy. This value constitutes the monopolist's opportunity cost of decreasing the quality of the antibiotic over time as its profits will be necessarily lower. Note that this value is said properly discounted at the rate $\beta(t)$ in the sense that it accounts for the discount rate $\delta$, but also for the generic industry's response to a change in antibiotic efficacy, weighted by its impact on the common pool of efficacy $\alpha \partial f_{B} / \partial w=\alpha c_{B} /\left(r_{B} w^{2}\right)$. The higher the pressure exerted by industry $B$ on the common pool, or the lower the value of efficacy, the lower is the weight attached by the monopolist to future profitability of antibiotic efficacy. 
The evolution of $\lambda(t)$ is ambiguous, at least at the beginning of the time horizon. Making use of (12) and the definition of $\beta(t)$ in conjunction with (8), we obtain:

$$
\dot{\lambda}(t)=-\frac{\partial \pi}{\partial w}+\beta(t) \int_{t}^{T} \frac{\partial \pi}{\partial w} \exp \left(-\int_{t}^{s} \beta(\tau) d \tau\right) d s .
$$

As the first term on the right-hand-side of the former equation is negative, while the second term is positive and converges to 0 as $t$ approaches $T$, we can state that the shadow price decreases towards the end of the patent life and reaches $\lambda(T)=0$ when the patent expires. We now turn to the controllable parameter $\alpha$, which represents the biological distance within the two antibiotics $A$ and $B$ and determines how antibiotic $B$ affects the common pool of the class's treatment efficacy as compared to antibiotic $A$. In developing the new antibiotic $A$, the monopolist choses this relative distance $\alpha$, which is characterized by necessary condition (10). Caculating $\partial H / \partial \alpha=-\lambda(t) f_{B}(t)$, we find for an interior solution $0<\alpha<\bar{\alpha}$ after rearranging condition (10):

$$
-\frac{\partial C}{\partial \alpha}=\int_{0}^{T} \lambda(t) f_{B}(w(t)) e^{-\delta t} d t
$$

When this last condition holds, the monopolist chooses $\alpha$ such that the marginal cost of innovating antibiotic $A$, i.e. the marginal cost of increasing its relative distance with respect to antibiotic $B$, equals the inter-temporal marginal benefit, properly discounted, of industry's $B$ avoided impact on the common pool resource, evaluated at the shadow price $\lambda(t)$. Whenever the marginal cost of innovation outweighs the marginal benefit, a corner solution applies such that $\alpha=\bar{\alpha}$, where we recall that $\bar{\alpha}$ corresponds to the minimal distance prescribed by law. Finally, the condition that assures the monopolist's investment to be profitable is given by $C\left(\alpha^{m}\right)<\int_{0}^{T} e^{-\delta t} \pi\left(w^{m}(t), f_{A}^{m}(t)\right) d t$, where $\left(w^{m}(t), f_{A}^{m}(t), \alpha^{m}\right)$ satisfies the conditions defined in equations (6) to (11). Whether this last condition holds or not is an empirical question, which however lies outside the scope of this paper.

We have made use of numerical simulations in order to address the outcome in both markets, as well as the choice of $\alpha .{ }^{6}$ Many parameter values are conceivable for this stylized

\footnotetext{
${ }^{6}$ In particular, time and state space, as well as the controllable parameter space for $\alpha$ were discretized
} 
model and have been analyzed. We will concentrate on the baseline parameters as given in the following table which will apply unless specified otherwise.

\begin{tabular}{|c|c|c|c|c|c|c|c|}
\hline$w_{0}$ & $r_{A}$ & $r_{B}$ & $c_{A}$ & $c_{B}$ & $\delta$ & $T$ & $\bar{\alpha}$ \\
\hline \hline 18.5 & 0.31 & 0.30 & 3 & 3 & 0.04 & 20 & 2 \\
\hline
\end{tabular}

Antibiotics $A$ and $B$ differ slightly as the willingness to pay for antibiotic $A$ is bigger than that of antibiotic $B$, which follows from $r_{A}>r_{B}$, while production costs are equal. This implies that $c_{A} / r_{A}<c_{B} / r_{B}$ in the baseline case, such that the production of antibiotic $A$ can be sustained at lower levels of antibiotic treatment effectiveness. Furthermore, we postulate the functional form for the innovation cost as $C(\alpha)=1 / \alpha$. Figure 1 shows the results for the baseline case. We find that the monopolist chooses $\alpha^{m}=1.09$ and sells at a decreasing rate given by the continuous line $f_{A}^{m}$. Treatment rates in market $B$ are initially higher than in market $A$, as the generic industry does not account for the cost of using up antibiotic effectiveness. Once the patent expires at $T=20$, a generic industry takes over in market $A$, such that an upwards jump occurs in the treatment rate in market $A$ and continues to evolve at a higher level than in market $B$ as given by equilibrium condition (3).

We do not find any numerical evidence for $\dot{\lambda}>0$. Rather, the level of the implicit value $\lambda$ increases as a response to a higher profitability of market $A$ which is either implied by an increase in $r_{A}$ and $w_{0}$ or a decrease in $c_{A}$. The level of $\lambda$ then must decrease at a faster rate in order to reach $\lambda(T)=0$ when the patent expires.

A dynamic comparative exercise reveals the intuitive result that the monopolist's treatment rate is increasing in $r_{A}$ and $w_{0}$ and decreasing in $c_{A}$. While the decreasing pattern of the treatment rate $f_{A}^{m}$ over time remains unaffected by an increase in $r_{A}$, it can differ as a response to changes in the initial levels of antibiotic treatment effectiveness and the average production cost. Consider Figure 2, which has been drawn for various values of $w_{0}$ and where $c_{A}=1.5$. Here, the treatment rate $f_{A}^{m}$ monotonically increases over time for relatively high values of $w_{0}$ and evolves non-monotonically when initial treatment effectiveness is low. In and a forward-backward sweep method was applied to solve for the necessary conditions characterizing the intertemporal objective in the monopoly and the social optimum case. For the forward-backward sweep method, see Lenhart and Workman (2007). 
such a case, $f_{A}^{m}$ first decreases and increases in a final phase. As shown in Appendix A, this situation arises when rate of decrease in the level of treatment effectiveness is lower than the rate of decrease of the full marginal cost.

Finally, we present the impact of a change in the patent length, $T$, and of the valuation parameter, $r_{A}$, on the monopolist's profit-maximizing choice of $\alpha^{m}$ in Figure 3. As can be awaited, a longer patent duration or higher willingness to pay in market $A$ increases the monopolist's profitability, such that a more distant antibiotic analogue is innovated, i.e. $\alpha^{m}$ decreases.

\subsection{Social optimum}

We now address how the new antibiotic analogue $A$ should be positioned and sold in conjunction with antibiotic $B$ from a social point of view. This will serve as a benchmark for comparison with the monopoly outcome just described. The social optimum to be characterized is not constrained by the minimum distance between antibiotics nor by the patent duration. For each market $A$ and $B$ we define the gross surplus from consumption as

$$
U_{i}\left(f_{i}, w\right)=\int_{0}^{f_{i}} r_{i} w(1-f) d f,
$$

which represents the surface below the price function $P_{i}\left(f_{i}, w\right)=\partial U_{i} / \partial f_{i}$. The intertemporal social objective then consists in:

$$
\begin{gathered}
\max _{\left\{\alpha, T_{A}, T_{B}, f_{A}, f_{B}\right\}} B E=\int_{0}^{T_{A}} e^{-\delta t}\left[U_{A}\left(f_{A}, w\right)-c_{A} f_{A}\right] d t-C(\alpha)+ \\
\qquad \int_{0}^{T_{B}} e^{-\delta t}\left[U_{B}\left(f_{B}, w\right)-c_{B} f_{B}\right] d t \\
\text { s.t. } \dot{w}(t)=-f_{A}-\alpha f_{B}, \quad w(0)=w_{0}, \quad w(T) \geq 0, \\
\alpha \geq 0,
\end{gathered}
$$

where the bracketed terms in (14) represent the net social surplus from consumption in market $i=A, B$, respectively. We also assume that the discount rate $\delta$ and the initially available stock, $w_{0}$, are identical to the monopoly case. 
A necessary condition for market $i=A, B$ to be served at time $t$ is that antibiotic $i$ is economically viable, i.e. $w(t) \geq c_{i} / r_{i}$. We point out here that the time when it is socially optimal to stop serving a market generally differs, i.e. $T_{A} \neq T_{B}$. Let $T^{*}=\max \left\{T_{A}, T_{B}\right\}$ be the point of time when it is socially desirable to stop using antibiotic $i$ characterized by the minimum level $c_{i} / r_{i}=\min \left\{c_{A} / r_{A}, c_{B} / r_{B}\right\}$.

We write the current-value Hamiltonian as:

$$
H=w\left[\int_{0}^{f_{A}} r_{A}(1-f) d f+\int_{0}^{f_{B}} r_{B}(1-f) d f\right]-c_{A} f_{A}-c_{B} f_{B}-z\left[f_{A}+\alpha f_{B}\right]
$$

where $z(t)$ is the implicit value associated to the common pool resource in the social optimum. The following conditions represent the necessary conditions for a social optimum:

$$
\begin{aligned}
f_{i} & \geq 0, \quad \frac{\partial H}{\partial f_{i}} \leq 0 \text { and } f_{i} \frac{\partial H}{\partial f_{i}}=0, \quad \text { for } i=A, B \\
f_{i} & \leq 1, \quad \frac{\partial H}{\partial f_{i}} \geq 0 \text { and }\left(1-f_{i}\right) \frac{\partial H}{\partial f_{i}}=0, \quad \text { for } i=A, B \\
-\frac{\partial H}{\partial w} & =-\left[\int_{0}^{f_{A}} r_{A}(1-f) d f+\int_{0}^{f_{B}} r_{B}(1-f) d f\right]=\dot{z}-\delta z, \\
\dot{w}(t) & =-f_{A}-\alpha f_{B},
\end{aligned}
$$

with $\frac{\partial H}{\partial f_{A}}=w r_{A}\left(1-f_{A}\right)-c_{A}-z$ and $\frac{\partial H}{\partial f_{B}}=w r_{B}\left(1-f_{B}\right)-c_{B}-\alpha z$, as well as the necessary condition for the controllable parameter

$$
\alpha \geq 0, \quad \alpha\left(\int_{0}^{T^{*}} e^{-\delta t} \frac{\partial H}{\partial \alpha} d t-\frac{\partial C}{\partial \alpha}\right)=0, \quad-\frac{\partial C}{\partial \alpha}+\int_{0}^{T^{*}} e^{-\delta t} \frac{\partial H}{\partial \alpha} d t \leq 0 .
$$

and transversality conditions

$$
w\left(T^{*}\right) \geq 0, \quad z\left(T^{*}\right) \geq 0 \quad \text { and } \quad w\left(T^{*}\right) z\left(T^{*}\right)=0,
$$

to which we add the additional condition related to the free terminal time $T^{*}$ :

$$
H\left(T^{*}\right)=0
$$

As in the monopoly case, it is not economically viable for $c_{i}>0$ to have $w\left(T^{*}\right)=0$. Consequently, we must necessarily have $w\left(T^{*}\right)>0$ and $z\left(T^{*}\right)=0$. 
Solving for $z(t)$ in the differential equation (18), we make use of the fact that $z\left(T^{*}\right)=0$ as a boundary condition and obtain:

$$
\begin{aligned}
z(t) & =\int_{t}^{T^{*}} e^{-\delta(s-t)}\left[\frac{\partial U_{A}}{\partial w}+\frac{\partial U_{B}}{\partial w}\right] d s \\
& =\int_{t}^{T^{*}} e^{-\delta(s-t)}\left[\int_{0}^{f_{A}} r_{A}(1-f) d f+\int_{0}^{f_{B}} r_{B}(1-f) d f\right] d s .
\end{aligned}
$$

The implicit value $z(t)$ thus represents the inter-temporal, discounted sum of the marginal surplus related to the antibiotic efficacy in the two markets. Stated differently, it represents the socially optimal opportunity cost associated to a decrease in the level of antibiotic efficacy in terms of social surplus. As in the monopoly case, we can only establish analytically that $z(t)$ eventually decreases towards the end of the time horizon and converges towards 0 when the economic viability of the last antibiotic has been extracted completely.

As the implicit value $z$ is positive and $c_{i}>0$, a corner solution $f_{i}=1$ cannot be socially optimal as the set of equations (17) does not hold. Using the inverse demand function given in (1) and rearranging the set of equations (16) for both markets $A, B$, we can write:

$$
\begin{aligned}
& P_{A} \leqq c_{A}+z, \quad \text { if }<\text { then } f_{A}(t)=0 \\
& P_{B} \leqq c_{B}+\alpha z, \quad \text { if }<\text { then } f_{B}(t)=0 .
\end{aligned}
$$

It is socially optimal to use antibiotic $i$, when its price is equal to its full marginal cost, where the latter accounts for the social cost of using the common pool's efficacy and is weighted by the antibiotic's relative pressure.

We calculate the difference in the socially optimal treatment rates, $f_{A}-f_{B}$, when both antibiotics are used simultaneously, i.e. $f_{A}>0$ and $f_{B}>0$ :

$$
f_{A}-f_{B}=\frac{1}{w}\left(\frac{c_{B}}{r_{B}}-\frac{c_{A}}{r_{A}}+z(t) \frac{\alpha r_{A}-r_{B}}{r_{A} r_{B}}\right) \lesseqgtr 0,
$$

The sign of $f_{A}-f_{B}$ depends on the model parameters, in particular the level of relative economic viability and valuation in each market, as well as on the controllable parameter $\alpha$ and the implicit value of antibiotic efficacy, $z(t)$. Consider the case that antibiotic $B$ is less 
economically viable as compared to antibiotic $A$, i.e. $\frac{c_{B}}{r_{B}}>\frac{c_{A}}{r_{A}}$, and that the relative pressure exerted by antibiotic $B$ on the common pool of efficacy guarantees $\alpha r_{A}<r_{B}$. In this case, the sign of the difference in the treatment rates may change. In particular, it can occur that $f_{A}-f_{B}<0$ during an early phase because the lower economic viability of antibiotic $B$ is outweighed by the relatively lower impact of antibiotic $B$ on the common pool of antibiotic efficacy. However, as $z(t)$ eventually decreases, the difference in economic viability levels becomes more and more important and eventually dominates, such that in a final phase, we must have $f_{A}-f_{B}>0$. Whenever economic viability is identical in both markets, $\frac{c_{B}}{r_{B}}=\frac{c_{A}}{r_{A}}$, only the second effect exists, such that either $f_{A}-f_{B}>0$ or $f_{A}-f_{B}<0$ depending on parameters related to the willingness to pay, $r_{A}, r_{B}$, and relative pressure $\alpha$ exerted by antibiotic $B$. The socially optimal choice of $\alpha$ is given by: $-\frac{\partial C}{\partial \alpha}=\int_{0}^{T^{*}} z(t) f_{B} e^{-\delta t} d t$, which indicates that the marginal cost of innovation is equal to the discounted inter-temporal social value of treatment effectiveness, weighted by the marginal impact of antibiotic $B$. Finally, we can use $z\left(T^{*}\right)=0$ in transversality condition (22) in order to find:

$$
U_{A}\left(f_{A}, w\right)-c_{A} f_{A}+U_{B}\left(f_{B}, w\right)-c_{B} f_{B}=0
$$

where we recall for $i=A, B$ that $U_{i}=\int_{0}^{f_{i}} r_{i} w(1-f) d f=r_{i} w f_{i}\left[1-f_{i} / 2\right]$. Consequently, equation (27) is satisfied when $f_{A}\left(T^{*}\right)=f_{B}\left(T^{*}\right)=0$. When $\frac{c_{A}}{r_{A}} \neq \frac{c_{B}}{r_{B}}$, the use of the less economically viable antibiotic stops already before $T^{*}$ is reached. However, when $\frac{c_{A}}{r_{A}}=\frac{c_{B}}{r_{B}}$, numerical evidence suggests that the treatment rates $f_{i}$ converge simultaneously to 0 which will occur when the level of antibiotic efficacy has reached $w\left(T^{*}\right)=c_{i} / r_{i}$.

Two cases are illustrated in Figure 4. The continuous and dashed lines correspond to the case of baseline parameters with $r_{A}=0.31>0.3=r_{B}$ and $c_{A}=c_{B}=3$ such that $c_{A} / r_{A}<c_{B} / r_{B}$. In the baseline case, antibiotic $B$ is used more intensively initially, but falls below the use of antibiotic $A$ at around $T=63$. The critical level of economic viability of antibiotic B will be reached first, while antibiotic $A$ remains economically viable in a final phase before its level of economic viability is reached. When both antibiotics have identical levels of economic viability, $c_{A} / r_{A}=c_{B} / r_{B}$, the dotted and dash-dotted lines apply. Both 
antibiotics are produced simultaneously up to $T^{*}$. Numerical evidence suggests in this case that antibiotic $B$ should be used more intensively because it exerts relatively less pressure on the common pool of treatment effectiveness as compared to antibiotic $A\left(\alpha^{*}<1\right)$.

Finally, numerical evidence suggests that an increase in $r_{A}$, while all other parameters remain at their baseline level, increases $\alpha^{*}$ and decreases $T^{*}$. The driving mechanism is that the use of antibiotic $B$ will decrease, and it is socially optimal to abandon market $B$ and concentrate on market $A$ (for values $r_{A} \geq 0.4$ ). Higher valuation in market $A$ then clearly leads to an earlier exhaustion of antibiotic effectiveness. The following table summarizes these results:

\begin{tabular}{|c|c|c|c|c|}
\hline$r_{A}$ & 0.3 & 0.31 & 0.35 & 0.4 \\
\hline \hline$\alpha^{*}$ & 0.365 & 0.385 & 0.435 & 2 \\
\hline$T^{*}$ & 290 & 253 & 161 & 136 \\
\hline \hline$\alpha^{m} \mid T=20$ & 1.227 & 1.09 & 0.85 & 0.695 \\
\hline
\end{tabular}

\section{Economic instruments}

Karp (1992a) and Karp (1992b) have looked at the welfare implications of economic agents exploiting a common pool resource in an oligopolistic context and have proposed economic instruments, like combined mechanisms of taxation and subsidy, which induce an efficient use. Although we build on that approach, the novelty of the economic instruments developed here relies on the fact that two different markets are served (either by a monopolist or a generic, competitive industry), that demand is state-dependent and that resource use in each market affects the common resource pool differently.

The economic instruments developed here can induce both markets to operate efficiently if the monopolist positions its antibiotic $\mathrm{A}$ in a socially optimal way, i.e. $\alpha^{m}=\alpha^{*}$. However, as the discounted sum of inter-temporal monopolistic profits related to market $A$ is clearly lower than the social surplus associated to both markets $A$ and $B$, the monopolist does not have the incentives to innovate as "far" as it would be socially optimal. As a consequence, a subsidy targeting the research and development of sufficiently differentiated products is 
warranted..$^{7,8}$

In market $B$, where a generic industry operates without attributing any implicit value to the common pool resource, a unitary tax set at a level

$$
\tau_{B}(t)=\alpha^{*} z(t)
$$

will induce the industry to produce at the socially-optimal level as market incentives (see equation (2)) and social optimum (see equation (25)) will coincide. In order to induce the monopolist to sell antibiotic $A$ at the socially optimal level, a tax-subsidy mechanism may be implemented. Bergstrom et al. (1981) propose a unitary, time-dependent, mechanism, in contrast to Karp and Livernois (1992) who propose a unitary, state-dependent mechanism. The state-dependent mechanism has the desirable property to be Markov-perfect and to prevent the potentially strategic behavior by the monopolist when facing a time-dependent mechanism.

For later reference, we start with the unitary, time-dependent tax or subsidy, $m_{A}(t)$, applied to sales of the antibiotic in market $A$. Assuming the cost of innovation to be sunk $\left(\alpha^{m}=\alpha^{*}\right)$, the monopolist's modified objective (omitting the constraints which remain unchanged) then becomes:

$$
\max _{\left\{f_{A}(t)\right\}} \int_{0}^{T} e^{-\delta t}\left[\pi\left(w(t), f_{A}(t)\right)+m_{A}(t) f_{A}(t)\right] d t+e^{-\delta T} V_{A}^{o a}(w(T)),
$$

and the corresponding static efficiency condition is

$$
\frac{\partial H}{\partial f_{A}}=\frac{\partial \pi(\cdot)}{\partial f_{A}}+m_{A}(t)-\lambda(t)=0
$$

which holds for an interior solution $0<f_{A}<1$. Making use of the characterization of the

\footnotetext{
${ }^{7} \mathrm{An}$ anonymous referee suggests that it may be impossible for the regulator to set up a subsidy implying $\alpha^{m}=\alpha^{*}$, in particular, when $\alpha$ cannot be measured adequately. In such a case, a second-best policy of economic instruments would have to be developed which minimizes the intertemporal social loss resulting from an inefficient level of innovation and antibiotic market supply.

${ }^{8}$ We do not claim that economic instruments are necessarily superior to other tools which a regulator may apply (e.g. restricting the use of particular antibiotics for a precise infection). However, we believe that economic instruments represent a good tool to prevent a misuse of antibiotics when they should be reserved for last resort treatment.
} 
implicit value $\lambda(t)$ given in equation (12), we can rearrange the last equation and obtain

$$
m_{A}^{*}(t)=-\frac{\partial \pi}{\partial f_{A}}+\underbrace{\int_{t}^{T} \frac{\partial \pi}{\partial w} \exp \left(-\int_{t}^{s} \beta(\tau) d \tau\right) d s}_{\equiv \lambda^{*}}
$$

where $\lambda^{*}$ is evaluated along the socially optimal values $f_{A}=f_{A}^{*}, f_{B}=f_{B}^{*}$ and $w=w^{*}$. The mechanism thus offsets the monopolist's market power (the first term on the right-hand-side of (31)) and sends the "corrected" signal with respect to the implicit value of the resource (the second term of the right-hand side of (31)). Note that this second term is increasing in the patent length, $T$, implying that a longer patent duration increases $m_{A}(t) .{ }^{9}$

The sign of $m_{A}^{*}(t)$ is not straightforward to determine analytically. We can state that $\lambda^{*} \geq 0$ implying that the monopolist can benefit from a non-negative rent associated to the resource pool, while the sign of $-\partial \pi / \partial f_{A}$ may be negative evaluated at $f_{A}=f_{A}^{*}$. In particular, whenever a tax should be levied on antibiotic sales $\left(m_{A}(t)<0\right)$, that tax will be lower the longer the patent duration, while a subsidy $\left(m_{A}(t)>0\right)$ will be higher the longer the remaining patent duration. We have implemented numerically the time-dependent mechanism given in (31). Depending on the model parameters, many different configurations for such a mechanism exist. Whether the mechanism turns out to be a pure tax, pure subsidy, or a tax-subsidy combination depends on whether the monopolist over- or underproduces antibiotic $A$ as compared to the social optimum.

Consider again the baseline parameter case, where $c_{A} / r_{A}<c_{B} / r_{B}$. In Figure 5 , we present the mechanism $m_{A}(t)$ for $t \in[0, T]$, where various patent durations have been considered $(T \in\{20,30,50,70,90,100,150\})$. For relatively short patent durations, the policy instrument turns out to be a tax, while for relatively long patent durations, a tax should be levied initially, followed by a subsidy. The fact that the instrument tends to be a subsidy in a final phase relies on the fact that for relatively long patent durations, it is socially optimal

\footnotetext{
${ }^{9}$ The observation that the level of the optimal time-dependent instrument depends on the remaining patent length suggests a further economic instrument, which is patent length itself. As it impacts on the antibiotic efficacy's implicit value assigned by the monopolist, a continualblack "optimal" adjustment of the patent length may induce a socially optimal implicit value for the resource. Using patent duration as an economic instrument to manage resistance problems has been put forward by Kades (2005), the economic analysis of which lies outside the scope of this paper.
} 
to produce antibiotic $A$ only, as treatment effectiveness tends to have fallen below the economic viability level of antibiotic $B$. At the beginning of the patent, while both antibiotics are economically viable, a tax tends to apply on antibiotic $A$ because it is socially optimal to use antibiotic $B$ more intensively. ${ }^{10}$

Karp and Livernois (1992) have pointed out that time-dependent mechanisms can only induce socially optimal behavior by the monopolist, if the regulator can commit to the announced mechanism. In the opposite case, a monopolist may deviate from the optimal path of extraction, forcing the regulator to adjust its policy, and "by choosing the correct 'deviation', the monopolist can benefit from the change it forces upon the government (p.222)." A linear, markov-perfect mechanism, as proposed by Karp and Livernois (1992), with total payments of the form $m_{A}(w) f_{A}$ precludes such strategic behavior because it is "subgame perfect." Replacing $m_{A}(t)$ in $(29)$ by $m_{A}(w)$ gives the monopolist's new objective when facing a state-dependent mechanism. Developing the necessary conditions which characterize the monopolist's solution, we find that the static and dynamic conditions now write for an interior solution $f_{A}$ as:

$$
\begin{aligned}
\lambda(t) & =\frac{\partial \pi(\cdot)}{\partial f_{A}}+m_{A}(w) \\
\dot{\lambda}(t)-\delta \lambda(t) & =-\frac{\partial \pi(\cdot)}{\partial w}-\frac{\partial m_{A}(w)}{\partial w} f_{A}+\alpha \lambda(t) \frac{\partial f_{B}(w)}{\partial w}
\end{aligned}
$$

As the monopolist's current choice of $f_{A}$ depends on the current level of antibiotic efficacy, we can write $f_{A}(t)=\mathcal{F}_{A}(w(t))$, where $\mathcal{F}_{A}$ is the optimal feedback rule followed by the monopolist. In order to characterize the mechanism $m_{A}(w)$ described by the system of (partial) differential equations (32) and (33), we first differentiate equation (32) with respect to time which yields

$$
\dot{\lambda}=\left(\frac{\partial^{2} \pi}{\partial f_{A}{ }^{2}} \frac{\partial \mathcal{F}_{A}}{\partial w}+\frac{\partial^{2} \pi}{\partial f_{A} \partial w}\right) \dot{w}+\frac{\partial m_{A}(w)}{\partial w} \dot{w}
$$

Substituting (32) and (34) into (33) allows to eliminate the co-state variable $\lambda$, and to find

\footnotetext{
${ }^{10}$ The reader may refer again to Figure 4 for the socially optimal evolution of treatment rates in the baseline case.
} 
a functional equation characterizing $m_{A}(w)$, which is:

$$
m_{A}(w)+\frac{\alpha f_{B}(w)}{\beta(w)} \frac{\partial m_{A}(w)}{\partial w}=-\frac{\partial \pi}{\partial f_{A}}+\frac{1}{\beta(w)}\left[\frac{\partial \pi}{\partial w}+\dot{w}\left(\frac{\partial^{2} \pi}{\partial f_{A} \partial w}+\frac{\partial^{2} \pi}{\partial f_{A}^{2}} \frac{\partial \mathcal{F}_{A}}{\partial w}\right)\right]
$$

where we recall the monopolist's endogenous discount rate, now defined with the argument $w$ as $\beta(w)=\delta+\alpha \partial f_{B}(w) / \partial w$.

In order to interpret this functional equation, we start with the simplest case where it is socially optimal to abandon market $B$, i.e. $f_{B}^{*}(t)=0, \forall t$, for which we have $\dot{w}=-\mathcal{F}_{A}$, such that equation (35) simplifies to

$$
\left.m_{A}^{*}(w)\right|_{f_{B}^{*}=0}=-\frac{\partial \pi}{\partial f_{A}}+\frac{1}{\delta}\left[\frac{\partial \pi}{\partial w}+\dot{w}\left(\frac{\partial^{2} \pi}{\partial f_{A} \partial w}+\frac{\partial^{2} \pi}{\partial f_{A}^{2}} \frac{\partial \mathcal{F}_{A}}{\partial w}\right)\right]
$$

where the terms on the right-hand side of equation (36) have to be evaluated along the socially optimal values $\mathcal{F}_{A}=f_{A}^{*}$ and $w=w^{*}$.

The first term on the right-hand side indicates that the state-dependent mechanism again offsets the monopolist's market power as is done by the time-dependent mechanism in equation (31). The second term on the right-hand-side is of particular interest, as it differs from the monopolist's corrected implicit value $\lambda^{*}$ (i.e. the corrected opportunity cost of decreasing antibiotic efficacy) included in the time-dependent mechanism (31). In particular, it does not depend on the patent duration or, stated differently, on the remaining patent life, a fact which emphasizes its state-dependent character. It can be interpreted as the present value of a (hypothetically constant) corrective incentive, which accounts for the marginal profitability of antibiotic efficacy, $\partial \pi / \partial w$, on the one hand, as well as for the efficacy's evolution, $\dot{w}$, and impact on marginal profits on the other hand. Running numerical simulations with $r_{A}=0.5$ while all other parameters take their baseline values, we find that $f_{B}^{*}(t)=0, \forall t$. Figure 6 shows that the state-dependent instrument is a subsidy, suggesting that the unregulated monopolist tends to underproduce antibiotic $A$ as compared to the social optmum. In Figure 6, we also represent the time-dependant instrument, $m_{A}(t)$, for various patent lengths. A shorter patent length (or remaining patent life) implies that the monopolist attaches a lower value to antibiotic efficacy, which tends to mitigate the 
underproduction by the monopolist such that lower subsidies are required. As it turns out, the time-dependent subsidy is always lower than $m_{A}^{*}(w)$, which implies that the monopolist can capture higher rents when facing a state-dependent instrument.

The form of the unit tax-subsidy mechanism which applies to the present model with $f_{B}^{*}>0$ can also be derived. As it turns out, the mechanism makes intervene again the - now corrected - endogenous discount rate $\beta\left(w^{*}\right)$ as well as the relative pressure exerted by market $B$ on the common pool of antibiotic efficacy, $\alpha f_{B}^{*}$. In order to find the general solution to equation (35), we rearrange it first as:

$$
\frac{\partial m_{A}(w)}{\partial w}+\frac{\beta(w)}{\alpha f_{B}(w)} m_{A}(w)=\underbrace{-\frac{\beta(w)}{\alpha f_{B}(w)} \frac{\partial \pi}{\partial f_{A}}+\frac{1}{\alpha f_{B}(w)}\left[\frac{\partial \pi}{\partial w} \dot{w}\left(\frac{\partial^{2} \pi}{\partial f_{A} \partial w}+\frac{\partial^{2} \pi}{\partial f_{A}{ }^{2}} \frac{\partial \mathcal{F}_{A}}{\partial w}\right)\right]}_{\equiv \Omega\left(w, f_{A}, f_{B}\right)},
$$

and define the integrating factor $\Gamma(w)=\exp \left[\int_{0}^{w} \frac{\beta(u)}{\alpha f_{B}(u)} d u\right]$, which leads to

$$
m_{A}^{*}(w)=[\Gamma(w)]^{-1} \int_{0}^{w} \Gamma(u) \Omega\left(u, f_{A}^{*}, f_{B}^{*}\right) d u
$$

where use has been made of $m_{A}^{*}(0)=0$ and where the terms on the right-hand side of equation (37) have again to be evaluated along the socially optimal values $\mathcal{F}_{A}=f_{A}^{*}, f_{B}=f_{B}^{*}$ and $w=w^{*}$. As the resource pool has to be optimally shared whenever $f_{A}^{*}, f_{B}^{*}>0$, the state-dependent policy will differ. As shown in Appendix B, the state-dependent policy is greater than the time-dependent one, when $\partial m_{A} / \partial w>0$. As the level of antibiotic efficacy is decreasing inevitably (as long as $f_{A}, f_{B}>0$ ), it goes in hand with a decrease in the state-dependent subsidy or increase in the state-dependent tax when $\partial m_{A} / \partial w>0$ holds. The monopolist anticipates the decrease in the level of antibiotic efficacy, and attaches relatively more value to the level of antibiotic efficacy when facing a state-dependent instrument $\left(\lambda_{s}(w(t))>\lambda_{o}(t)\right.$; for a proof, see Appendix B). Combining equations (30) and (32) evaluated along the socially optimal values (for the treatment rates and antibiotic efficacy), it follows that $m_{A}^{*}(w(t))>m_{A}(t)$, which states that taxes will be lower and subsidies will be higher with a state-dependent instrument as compared to a time-dependent one. This result occurs because of the interdependency of the monopolist's implicit value for antibiotic efficacy 
and the anticipated evolution of the state-dependent economic instrument as determined by the regulator (which does not exist in the context of a a time-dependent instrument). The opposite result holds when $\partial m_{A} / \partial w<0$.

\section{Concluding remarks}

We have modeled a monopolist's incentives of innovation and use of a new antibiotic belonging to a class of existing antibiotics and compared the outcome to the social optimum. Innovation occurs deterministically in the sense that the innovating firm can decide on the biological distance between its drug and an already existing one which is sold by a generic industry. The biological distance, or positioning, of the new drug determines how the already existing antibiotic affects a pool of antibiotic efficacy which is common to both antibiotics. We have addressed the simple case where each antibiotic serves a particular market. In particular, markets are only related by the fact that antibiotic efficacy is a quality indicator for each antibiotic, while antibiotics do not represent substitutes in use.

Our analysis has shown that the monopolist's opportunity cost of marginally distancing his drug from the existing one is given by the discounted, intertemporal sum of avoided impacts by the generic industry, valued at the shadow price of antibiotic efficacy. The shadow price of antibiotic efficacy as perceived by the monopolist captures the intertemporal value, properly discounted, of the antibiotic efficacy's marginal profitability. The monopolist's proper discount rate accounts for his pecuniary discount rate, as well as the generic industry's marginal pressure exerted on the common pool of antibiotic efficacy. The closer the antibiotics, the higher the relative pressure exerted by the generic industry, implying that lower weight is attached by the monopolistic firm to its future profits. The socially optimal incentives account for the marginal impact of antibiotic efficacy on both markets and do not make intervene an adjusted discount factor.

Market incentives of antibiotic use also differ from the social optimum and warrant the application of corrective instruments, such as a combined tax and subsidy mechanism. We 
present a time- and a state-dependent mechanism targeting the market served by the monopolistic firm. The mechanism offsets the monopolist's market power, corrects its implicit value associated to antibiotic efficacy, and, when state-dependent, prevents the monopolist from deviating strategically. We also calculate the time-dependent tax that must apply on the generic market when the monopolist is subject to the described tax-subsidy mechanism.

Our analysis on corrective economic instruments assumes that the monopolist innovates at the socially optimal distance from the existing drug which can be guaranteed by setting the biological minimum distance appropriately or by introducing an additional R\&D subsidy enhancing the biological distance chosen. Only when this assumption is fulfilled, will our proposed economic instruments correct both markets in conjunction. In the context of antibiotic drugs which are related to a common pool of antibiotic treatment efficacy, this points to the importance that warranting patents must not be guided by the marginal novelty of products only, but also account for their related impact on that common pool.

Future research may look at efficiency-inducing economic instruments in a context where the positive effect of antibiotic use on containing future prevalence of infection is accounted for. The socially optimal breadth and length of a patent conferred to antibiotic analogues also merits attention. A general model should address the possibility that antibiotics represent substitutes for a given infection. 


\section{References}

Becker, D., M. Selbach, C. Rollenhagen, M. Ballmaier, T.F. Meyer, M. Mann and D. Bumann (2006). Robust salmonella metabolism limits possibilities for new antimicrobials. Nature, 440: 303-307.

Coates, A.R.M., Halls, G. and Y. Hu (2011). Novel classes of antibiotics or more of the same? British Journal of Pharmacology, 163(1): 184-194.

EU directive 2001/82/EC, article 35, Annex 1, http://ec.europa.eu.

Fischer, C. and R. Laxminarayan (2004). Monopoly Extraction of an Exhaustible Resource with two markets. Canadian Journal of Economics, 37(1): 178-188.

Fischer, C. and R. Laxminarayan (2005). Sequential development and exploitation of an exhaustible resource: do monopoly rights promote conservation? Journal of Environmental Economics and Management, 49(3): 500-515.

Herrmann, M. (2010). Monopoly Pricing of an Antibiotic Subject to Bacterial Resistance. Journal of Health Economics, 29 (1): 137-150

Herrmann, M. and G. Gaudet (2009).The Economic Dynamics of Antibiotic Efficacy under Open Access, Journal of Environmental Economics and Management, 57: 334-350.

Herrmann, M. and R. Laxminarayan (2010). Antibiotic Effectiveness: New Challenges in Natural Resource Management. Annual Reviews of Resource Economic, 2: 125-139.

Kades, E. (2005). Preserving a Precious Resource: Rationalizing the Use of Antibiotics. Northwestern University Law Review, 99: 611-674.

Karp, L. (1992a). Efficiency Inducing Tax for a Common Property Oligopoly. The Economic Journal, 102(411): 321-332.

Karp, L. (1992b). Social Welfare in a Common Property Oligopoly. International Economic Review, 33(2): 353-372.

Karp, L. and J. Livernois (1992). On efficiency-inducing taxation for a non-renewable resource monopolist, Journal of Public Economics, 49: 219-239.

Laxminarayan, R. and G.M. Brown (2001). Economics of Antibiotic Resistance: A 
Theory of Optimal Use, Journal of Environmental Economics and Management, 42: 183206.

Laxminarayan, R. (2002). How Broad Should the Scope of Antibiotics Patents Be? American Journal of Agricultural Economics, 84(5): 1287-1292.

Lenhart, S. and J.T. Workman (2007). Optimal Control Applied to Biological Models. London: Chapman \& Hall / CRC.

Levy, S.B. (1992). The Antibiotic Paradox. New York and London: Plenum Press.

Mechoulan, S. (2007). Market structure and communicable diseases, Canadian Journal of Economics, 40: 468-492.

Nelson, J.M., T.M. Chiller, J.H. Powers, F.J. Angulo (2007). Fluoroquinolone-resistant Campylobacter species and the withdrawal of fluoroquinolones from use in poultry: a public health success story. Clinical Infectious Diseases, 44 (7): 977-980.

Rudholm, N. (2002). Economic implications of antibiotic resistance in a global economy. Journal of Health Economics, 21(6): 1071-1083.

US Food and Drug Administration, press release P05-48 published on July 28, 2005. Website http://www.fda.gov/NewsEvents/Newsroom/PressAnnouncements/2005/ucm108467.htm consulted on February 18, 2013.

Wilen, J.E. and S. Msangi (2003). Dynamics of Antibiotic Use: Ecological versus Interventionist Strategies to Manage Resistance to Antibiotics, in R. Laxminarayan (ed.), Battling Resistance to Antibiotics and Pesticides: An Economic Approach. Washington, DC: Resources for the Future: 17-41. 


\section{Appendix}

\section{A Evolution of $f_{A}$}

We recall the static efficiency condition for an interior solution of antibiotic production by the monopolist $\left(0<f_{A}<1\right)$, which is given by:

$$
r_{A} w\left(1-2 f_{A}\right)=c_{A}+\lambda
$$

In order to characterize the evolution of antibiotic use over time, we differentiate and rearrange the former equation to get:

$$
\begin{aligned}
\dot{f}_{A} & =\frac{1}{2 r_{A} w}\left[r_{A}\left(1-2 f_{A}\right) \dot{w}-\dot{\lambda}\right] \\
& =\frac{1}{2 r_{A} w}\left[\frac{c_{A}+\lambda}{w} \dot{w}-\dot{\lambda}\right],
\end{aligned}
$$

where use has been made of the static efficiency condition to obtain the last equality. It then follows that

$$
\dot{f}_{A}>(<) 0 \Leftrightarrow \frac{\dot{w}}{w}>(<) \frac{\dot{\lambda}}{c_{A}+\lambda} .
$$

As $\dot{w}(t) \leq 0, \forall t \in[0, T]$, and $\dot{\lambda}(t)<0$ at least in a final phase when $\lambda(t)$ converges to 0 , we can state that antibiotic production by the monopolist increases if the treatment effectiveness of the antibiotic class decreases at a lower rate than the full marginal cost. The contrary may occur if the pressure exerted on the common pool resource is important such that the treatment effectiveness decreases at a higher rate. Finally, if the implicit value $\lambda$ were to increase, which cannot be excluded in an initial phase, the antibiotic production by the monopolist necessarily decreases. 


\section{B Comparison of $m_{A}^{*}(t)$ and $m_{A}^{*}(w)$}

Depending on whether $\frac{\partial m_{A}}{\partial w}>0$ or $\frac{\partial m_{A}}{\partial w}<0$, we can compare the state-dependent with the time-dependent policy when treatment rates and antibiotic efficacy are at the socially

optimal level. Assume that $\frac{\partial m_{A}}{\partial w}>0$ and recall the monopolist's modified dynamic efficiency conditions which apply in each case:

$$
\begin{aligned}
& \dot{\lambda}_{s}-\beta(t) \lambda_{s}=-\frac{\partial \pi}{\partial w}-\frac{\partial m_{A}}{\partial w} f_{A}, \\
& \dot{\lambda}_{o}-\beta(t) \lambda_{o}=-\frac{\partial \pi}{\partial w},
\end{aligned}
$$

where $\lambda_{s}$ and $\lambda_{o}$ represent the monopolist's shadow price attached to antibiotic efficacy facing the state-dependent and time-dependent policies, respectively and where $\beta(t)=\delta+$ $\alpha^{*} \frac{d f_{B}}{d_{w}}\left(w^{*}(t)\right)$.

When $\frac{\partial m_{A}}{\partial w}>0$, it follows from (38) and (39) that $\dot{\lambda}_{s}-\beta(t) \lambda_{s}<\dot{\lambda}_{o}-\beta(t) \lambda_{o}$. Multiplying side by side this equation by the integrant factor $J(t)=\exp \left(\int_{t}^{T} \beta(s) d s\right)$, we derive

$$
\frac{d \lambda_{s}(t) J(t)}{d t}<\frac{d \lambda_{o}(t) J(t)}{d t}
$$

Integrating and using the fact that $\lambda_{o}(T)=\lambda_{s}(T)=0$, this inequality leads us to $\lambda_{o}(t)<$ $\lambda_{s}(t)$. It then follows that $m_{A}^{*}\left(w^{*}(t)\right) \equiv \lambda_{s}(t)-\frac{\partial \pi}{\partial f_{A}}(t)>m_{A}^{*}(t) \equiv \lambda_{0}(t)-\frac{\partial \pi}{\partial f_{A}}(t)$.

A similar argument applies for $\frac{\partial m_{A}}{\partial w}<0$ to get $m_{A}^{*}\left(w^{*}(t)\right)<m_{A}^{*}(t)$. 


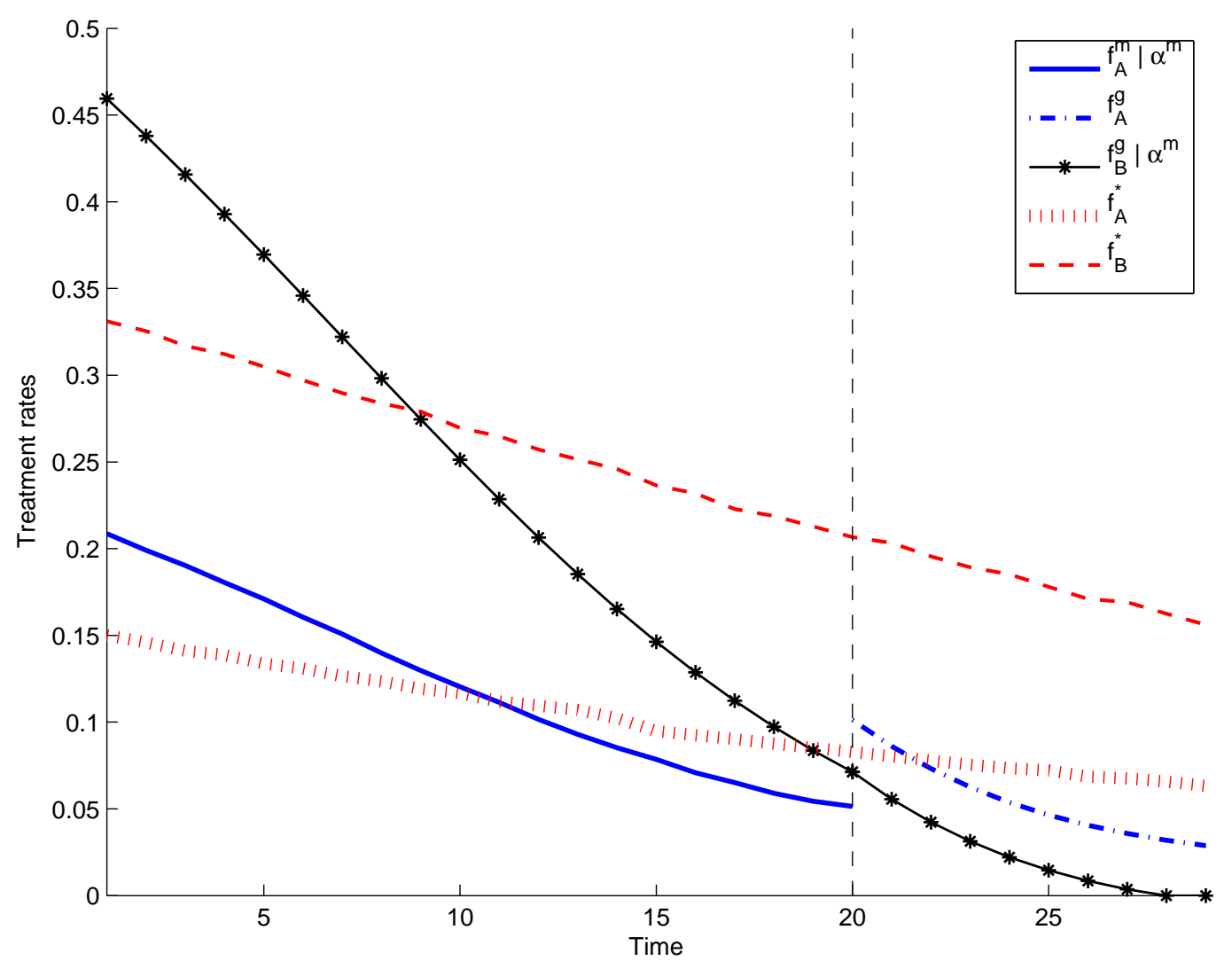

Figure 1: Market outcome in the baseline case 


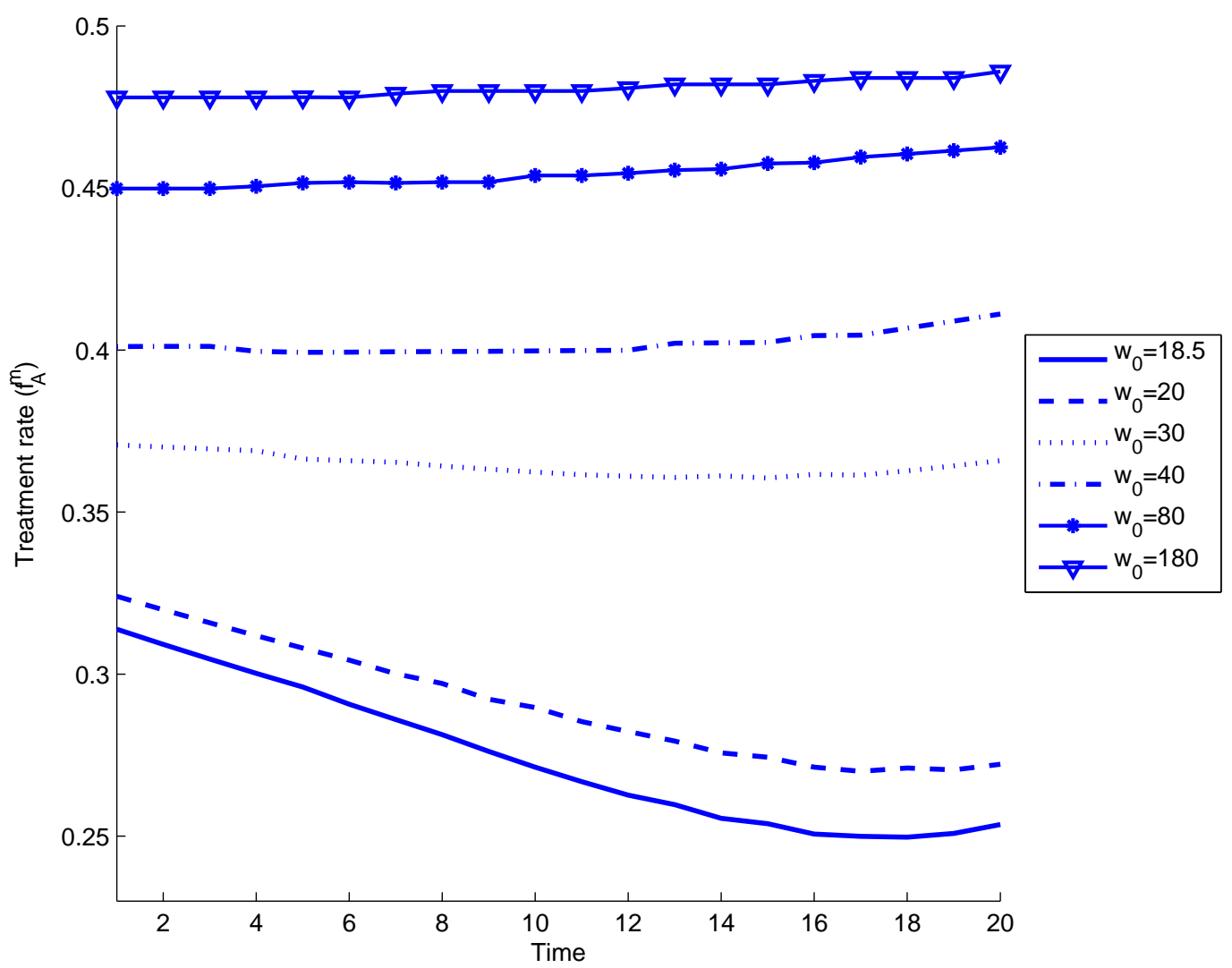

Figure 2: Dynamic comparative exercise on $w_{0}\left(\right.$ with $\left.c_{A}=1.5\right)$ 


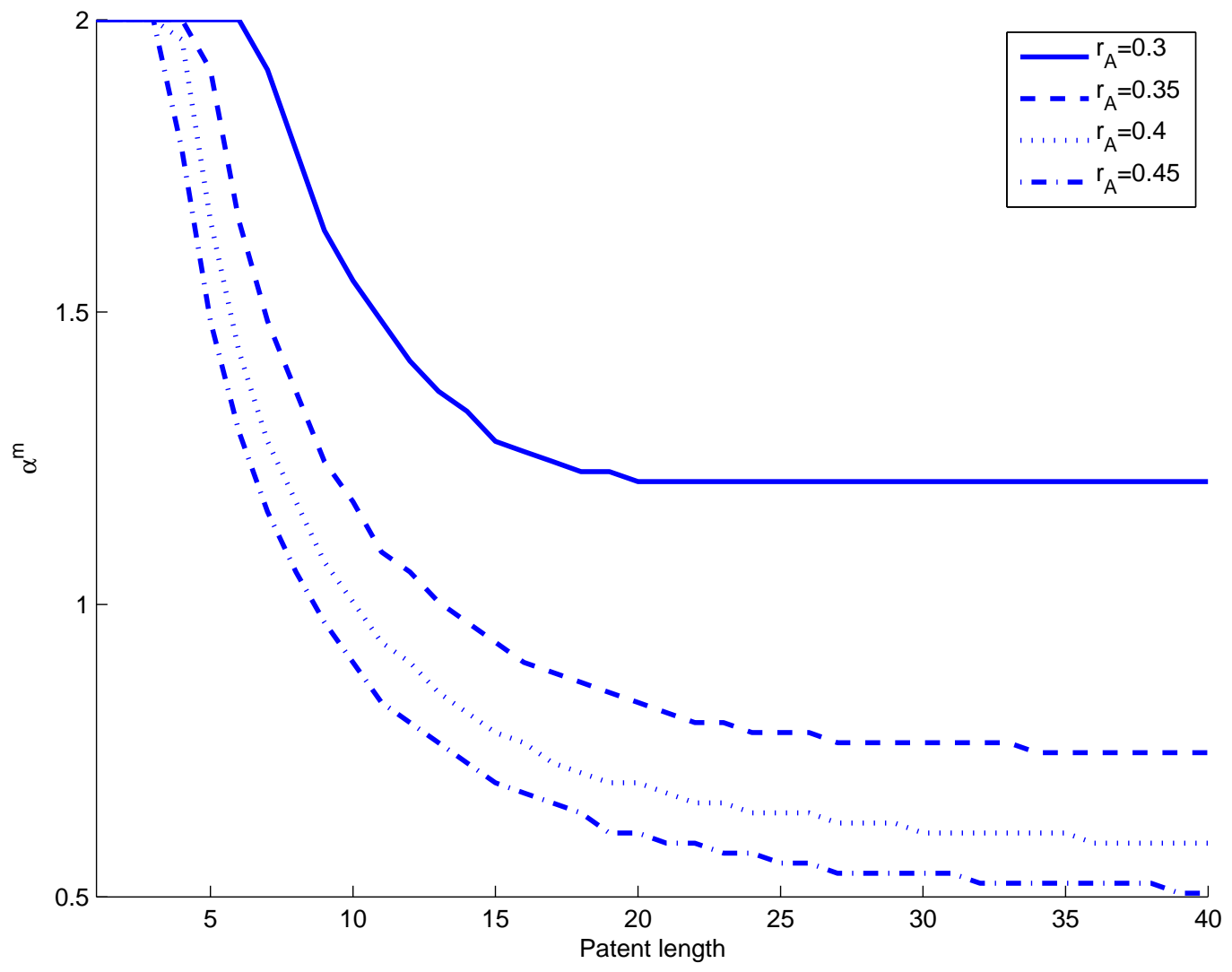

Figure 3: Dynamic comparative exercise on $T$ and $r_{A}$ 


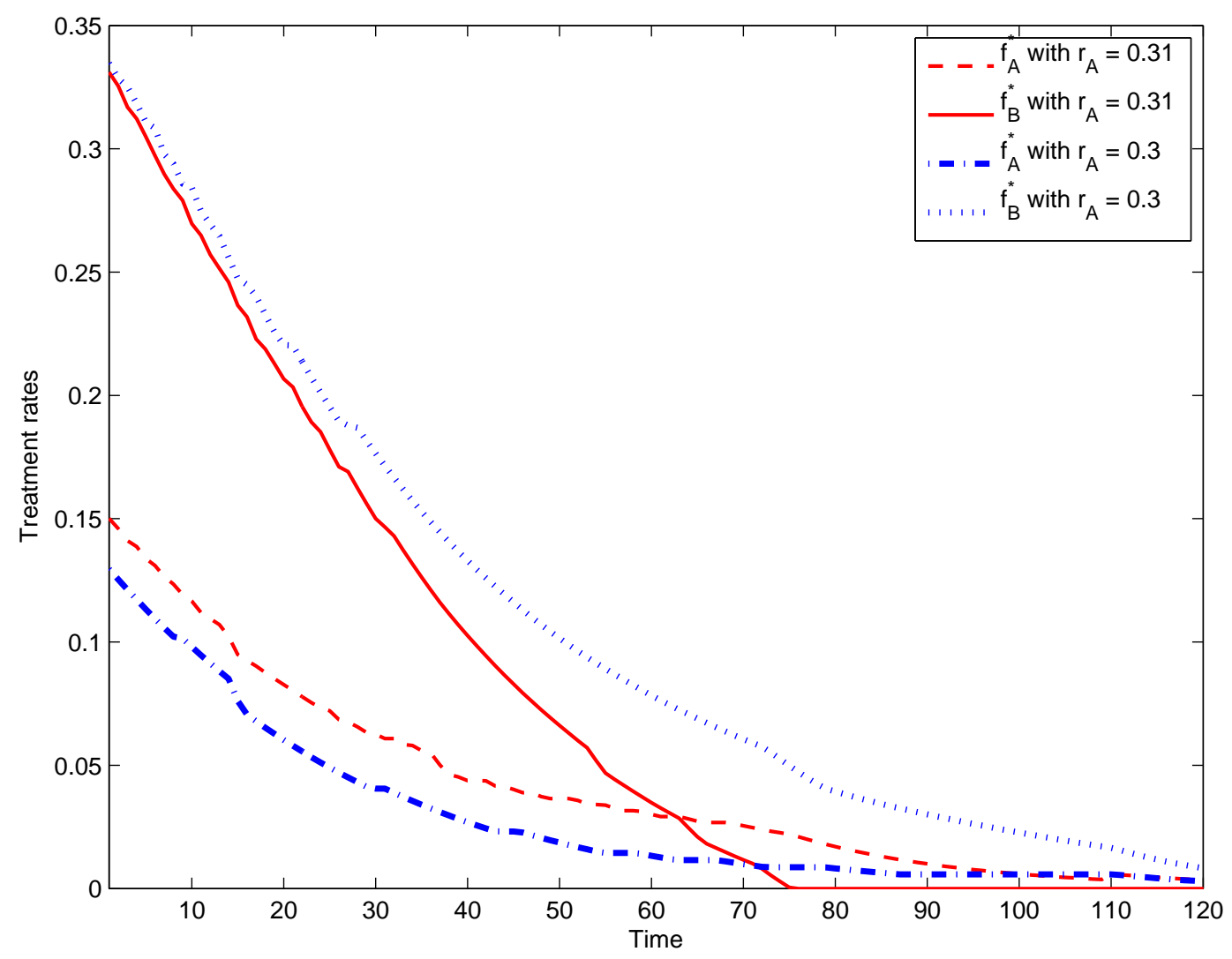

Figure 4: Socially optimal treatment rates $\left(f_{A}^{*}, f_{B}^{*}\right)$ 


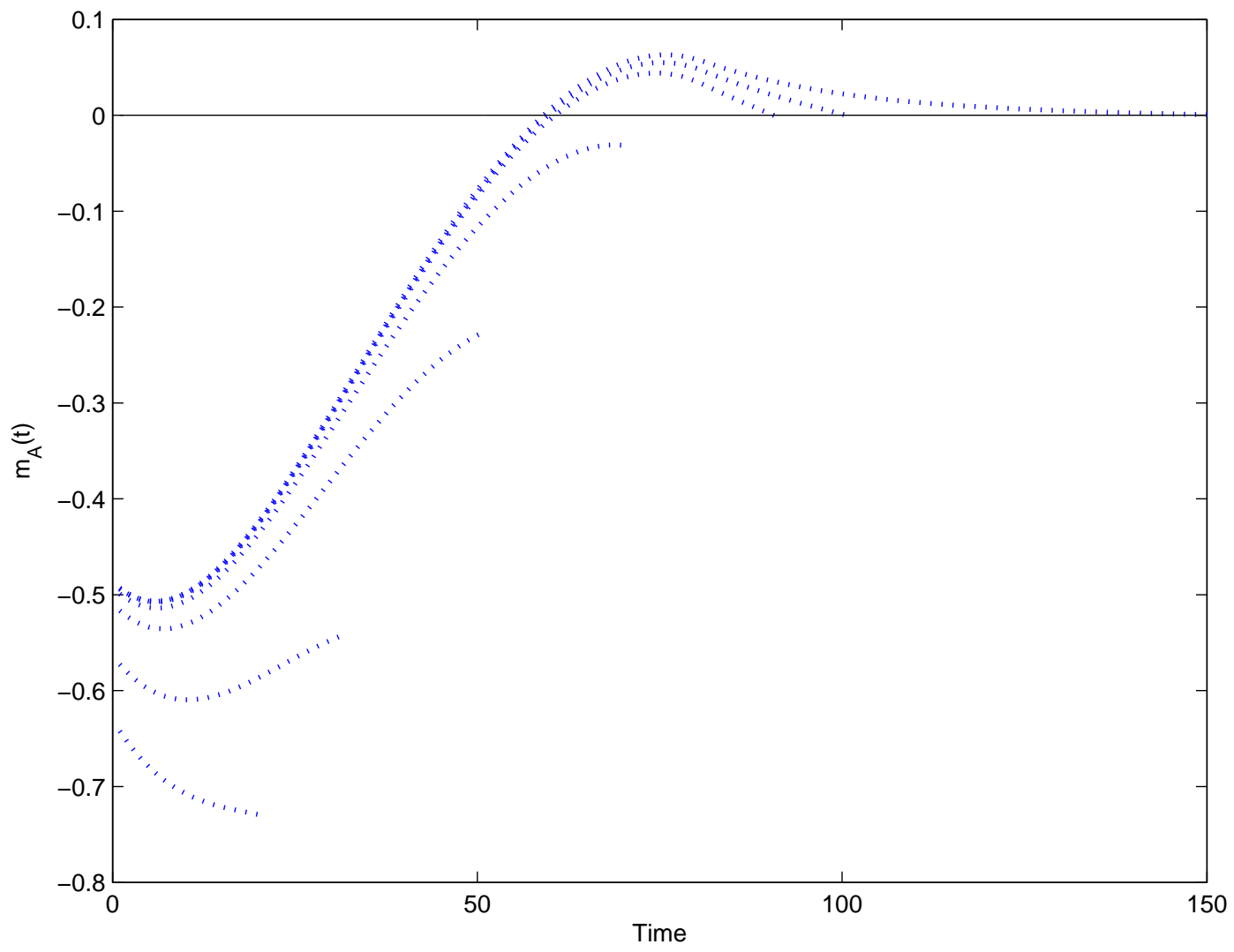

Figure 5: Time-dependent mechanism (baseline parameter values) 


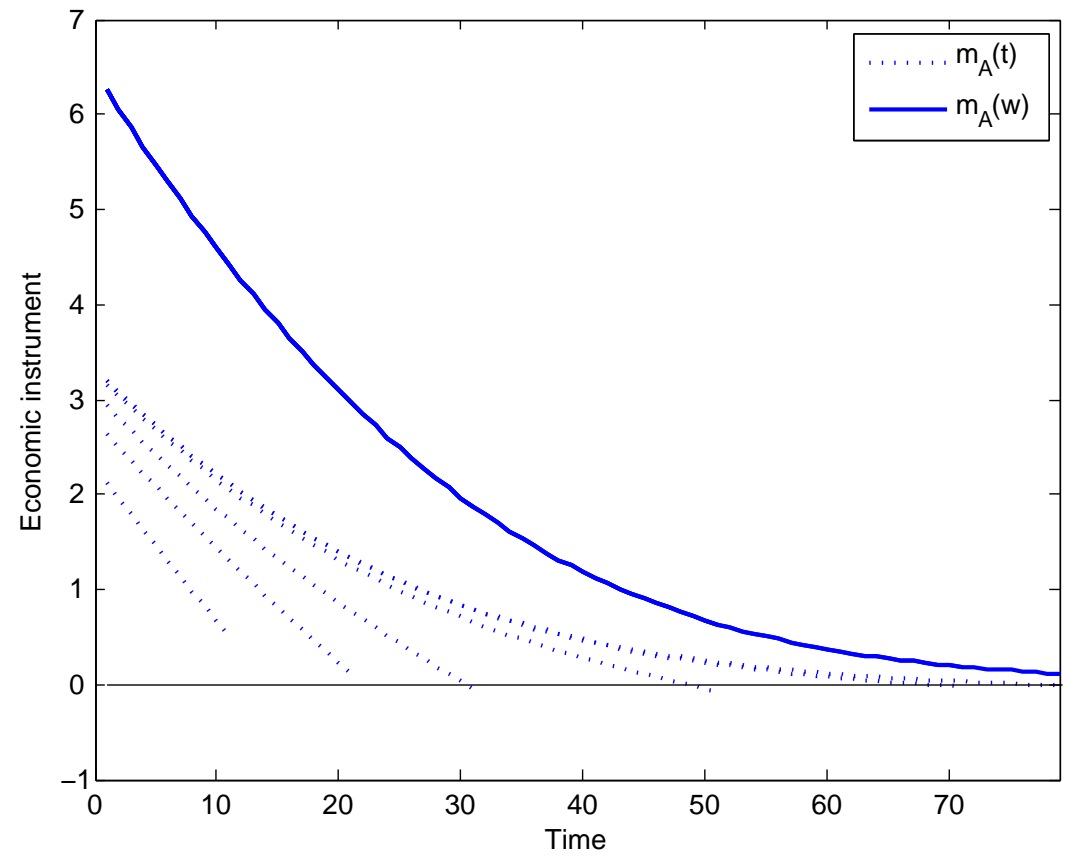

Figure 6: State-dependent mechanism $\left(r_{A}=0.5\right)$ 